\title{
STATISTICAL ANALYSIS ON THE ANTI- MICROBIAL EFFECT OF SWEET POTATO AND GRAPE LEAF EXTRACTED AGAINST SOME PATHOGENIC MICRO-ORGANISMS
}

\author{
Emiola, Olawale K.S. \\ Department of Statistics \\ Federal Polytechnic, Offa, Kwara State, Nigeria. \\ Omoloye, Musibau A. \\ Department of Statistics \\ Federal Polytechnic, Offa, Kwara State, Nigeria.
}

\begin{abstract}
Sweet potato and Grape leaf have been extensively used in medicine. The present investigation was undertaken to statistically evaluate the antimicrobial activity of these leaves extracts (methanolic and ethanolic) to determine the most significant extract and concentration that will inhibit the growth of some micro-organisms causing some certain diseases in the human system. The experiment was conducted on two species of microorganism (Staphylococcus aureus and Escherichia coli), each applied 5 different treatments; $18 \mathrm{mg} / \mathrm{ml}, 16 \mathrm{mg} / \mathrm{ml}$, $14 \mathrm{mg} / \mathrm{ml}, 12 \mathrm{mg} / \mathrm{ml}$ and $10 \mathrm{mg} / \mathrm{ml}$ of each extract. A randomized complete block design (two way ANOVA) was used to analyze the data (zone of inhibition) collected, while Duncan multiple range tests was used for the Post-hoc test (with the aid of Minitab 16 and SPSS 20). Result revealed that treatment $A$ extract with $18 \mathrm{mg} / \mathrm{ml}$ concentration is the best in inhibiting the growth of the micro-organisms in this study.
\end{abstract}

Keywords-Sweet potato, Grape, Staphylococcus aureus, Escherichia coli, ANOVA

\section{INTRODUCTION}

It was confirmed that plants are sources of nutrients and other bioactive moieties [3], responsible for vital functions in humans, therefore possess numerous health prospects [8], [13]. Energy and dietetic regimes linkages were reported in literature; also, the extraction of bioactive moieties and their useful on human metabolism need systematic and coherent which need further investigations, for establishment of meticulous and persuasive association for consumers [13].

Grape (Vitis vinifera $\mathrm{L}$.) was a leading fruit crop producing 70 million metric tons grapes every year in the world with extraordinary taste and flavor [11]. Various food products were produced from its fruit, seeds and leaves were used for

\author{
Abbah, Enoch A. \\ Department of Science Laboratory Technology \\ Federal Polytechnic, Offa, Kwara State, Nigeria. \\ Oladapo Asimiyu O. \\ Department of Computer and Mathematical Science \\ Osun State University, Osogbo, Osun State, Nigeria.
}

production of herbal medicines, as well as dietary supplements $[2,9]$.

Sweet potato (Ipomoea batatas L.) is a member of morning glory family (Convolvulaceae) [14] Sweet potato is among the major food crop in the world and is cultivated in all tropical and subtropical regions particularly in Asia, Africa and Pacific [4]. Asia and Africa accounts for 95 percent of the production [5]. The crop is mainly grown for tubers, but a large volume of sweet potato vines (Stems and Leaves) are left after harvesting [9]. The vines can be used as an alternative feed resource for ruminants to reduce competition between animals and humans for grains that is, maize and soya bean [10]. The value of sweet potato vines as feed supplement is enriched by high palatability [7], moderate level of crude protein and water soluble carbohydrate [12], and high level of digestibility greater than $62 \%$ [6],[1]

\section{MATERIALS AND METHODS}

Freshly young leaves of Sweet potato and Grape were collected from a tree during hamathan period of 2018, in the Federal Polytechnic Offa, and authenticated by Mr. Abbah, E.A. Lecturer in the Biology/Micro-Biology unit, Department of Science Laboratory Technology, Federal Polytechnic Offa, Offa Kwara State, Nigeria, 30 grams of the powdered plant materials were extracted successively with $600 \mathrm{ml}$ of methanol and ethanol as solvents for 4 days by Rotary evaporator equipment.

\section{A. Tests organisms-}

The test organisms used were clinical strain of bacteria which are pure culture of gram negative and gram positive bacteria respectively. They are Staphylococcus aureus and Escherichia coli respectively, they were propagated and stored in slant McCartney bottle at $4^{\circ} \mathrm{c}$ before use. 


\section{B. Experimentation-}

The agar diffusion method is used for the antimicrobial evaluations. The plates were sterilized in a hot air oven at $161^{\circ} \mathrm{c}$ for $2 \mathrm{hrs}$. After the preparation of different volume of each extract, it was applied into each well of the agar containing various isolates of bacteria in the plate to give their corresponding zone of inhibition. The diameter of the inhibition zone was measured in mm. An extract was classified as active when the diameter of the inhibition was equal to or larger than $8 \mathrm{~mm}$. All the run of the experiment were performed completely in randomized manner in Biology/Micro-biology laboratory, Science Laboratory Technology Department, the Federal Polytechnic Offa.

\section{Method of analysis}

The statistical tool used in the analysis is Randomized Complete Block Design (two way classification analysis of variance) to determine if there is any significant difference between the treatments (concentrations) and the plant extract, and Duncan multiple range test was also used to know which of these treatments and the plant extract is highly significant (if difference exist in the prior analysis).

Table 1: ANOVA table for Randomized Complete Block Design

\begin{tabular}{|c|c|c|c|c|}
\hline $\begin{array}{c}\text { Sources of } \\
\text { Variance }\end{array}$ & $\begin{array}{c}\text { Degree } \\
\text { of } \\
\text { freedo } \\
\mathrm{m}\end{array}$ & $\begin{array}{c}\text { Sum of } \\
\text { Squares }\end{array}$ & $\begin{array}{c}\text { Mean } \\
\text { Squares }\end{array}$ & $F_{0}$ \\
\hline $\begin{array}{c}\text { Treatment } \\
\mathrm{s}\end{array}$ & $a-1$ & $S S_{\text {Treatmen }}$ & $\frac{S S_{\text {Treatments }}}{a-1}$ & $\frac{M S_{\text {Treatme }}}{M S_{\text {Error }}}$ \\
\hline Blocks & $b-1$ & $S S_{\text {Blocks }}$ & $\frac{S S_{\text {Blocks }}}{b-1}$ & \\
\hline Errors & $\begin{array}{c}(a \\
-1)(b\end{array}$ & $S S_{\text {Error }}$ & $\frac{S S_{\text {Error }}}{(a-1)(b-1)}$ & \\
\hline Total & $N-1$ & & & \\
\hline
\end{tabular}

Statistical model: $y_{i j}=\mu+\tau_{i}+\beta_{j}+\epsilon_{i j}\left\{\begin{array}{l}i=1,2, \ldots, a \\ j=1,2, \ldots, b\end{array}\right.$

The hypotheses of interest are:

i. $H_{0}$ : There is no significant difference between the treatments

$H_{1}$ : There is significant difference between the treatments

ii. $H_{0}$ : There is no significant difference between the plant extracts

$H_{1}$ : There is significant difference between the plant extracts

Level of significance: 0.05

\section{EXPERIMENTAL AND RESULTS}

The antimicrobial activities of methanol and ethanol extracted from the leaves of Sweet potato and Grape against two pathogenic micro-organisms; such as Staphylococcus aureus and Escherichia coli were presented below.

Table 2: Methanolic extracted from sweet Potato leaf zone of inhibition (mm)

\begin{tabular}{|c|c|c|c|c|c|c|}
\hline \multirow[b]{2}{*}{$\begin{array}{c}\text { Organ } \\
\text { isms }\end{array}$} & \multicolumn{5}{|c|}{ Treatments } & \multirow[b]{2}{*}{ Total } \\
\hline & $\begin{array}{c}\text { A } \\
(18 \mathrm{mg} \\
/ \mathrm{ml})\end{array}$ & $\begin{array}{c}\mathrm{B} \\
(16 \mathrm{~m} \\
\mathrm{g} / \mathrm{ml})\end{array}$ & $\begin{array}{c}\mathrm{C} \\
(14 \mathrm{~m} \\
\mathrm{g} / \mathrm{ml})\end{array}$ & $\begin{array}{c}\mathrm{D} \\
(12 \mathrm{~m} \\
\mathrm{g} / \mathrm{ml})\end{array}$ & $\begin{array}{c}\mathrm{E} \\
(10 \\
\mathrm{mg} / \\
\mathrm{ml})\end{array}$ & \\
\hline $\begin{array}{c}\text { Staphy } \\
\cdot \\
\text { aureus }\end{array}$ & 24 & 22 & 18 & 16 & 12 & 92 \\
\hline E. coli & 26 & 20 & 17 & 15 & 10 & 88 \\
\hline Total & 50 & 42 & 35 & 31 & 22 & 180 \\
\hline Mean & 25.0 & 21.0 & 17.5 & 15.5 & 11.0 & \\
\hline
\end{tabular}

Table 3: Ethanolic extracted from sweet Potato leaf zone of inhibition (mm)

\begin{tabular}{|c|c|c|c|c|c|c|}
\hline \multirow[b]{2}{*}{$\begin{array}{c}\text { Organi } \\
\text { sms }\end{array}$} & \multicolumn{5}{|c|}{ Treatments } & \multirow[b]{2}{*}{ Total } \\
\hline & $\begin{array}{c}\mathrm{A} \\
(18 \mathrm{~m} \\
\mathrm{g} / \mathrm{ml})\end{array}$ & $\begin{array}{c}\mathrm{B} \\
(16 \mathrm{~m} \\
\mathrm{g} / \mathrm{ml})\end{array}$ & $\begin{array}{c}\mathrm{C} \\
(14 \mathrm{mg} / \\
\mathrm{ml})\end{array}$ & $\begin{array}{c}\mathrm{D} \\
(12 \mathrm{~m} \\
\mathrm{g} / \mathrm{ml})\end{array}$ & $\begin{array}{c}\mathrm{E} \\
(10 \mathrm{~m} \\
\mathrm{g} / \mathrm{ml})\end{array}$ & \\
\hline $\begin{array}{c}\text { Staphy. } \\
\text { aureus }\end{array}$ & 18 & 16 & 14 & 10 & 8 & 66 \\
\hline E. coli & 20 & 18 & 15 & 12 & 9 & 74 \\
\hline Total & 38 & 34 & 29 & 22 & 17 & 140 \\
\hline Mean & 19 & 17 & 14.5 & 11 & 8.5 & \\
\hline
\end{tabular}

Table 4: Methanolic extracted from Grape leaf zone of inhibition (mm)

\begin{tabular}{|c|c|c|c|c|c|c|}
\hline \multirow{3}{*}{$\begin{array}{c}\text { Orga } \\
\text { nism } \\
\text { s }\end{array}$} & $\begin{array}{c}\mathrm{A} \\
(18 \mathrm{~m} \\
\mathrm{g} / \mathrm{ml})\end{array}$ & $\begin{array}{c}\mathrm{B} \\
(16 \mathrm{mg} / \\
\mathrm{ml})\end{array}$ & $\begin{array}{c}\mathrm{C} \\
(14 \mathrm{~m} \\
\mathrm{g} / \mathrm{ml})\end{array}$ & $\begin{array}{c}\mathrm{D} \\
(12 \mathrm{mg} / \\
\mathrm{ml})\end{array}$ & $\begin{array}{c}\mathrm{E} \\
(10 \mathrm{mg} / \\
\mathrm{ml})\end{array}$ & Total \\
\hline $\begin{array}{c}\text { Staph } \\
y . \\
\text { aure } \\
\text { us }\end{array}$ & 26 & 21 & 19 & 15 & 12 & 93 \\
\hline $\begin{array}{c}\text { E. } \\
\text { coli }\end{array}$ & 29 & 26 & 20 & 18 & 14 & 107 \\
\hline Total & 55 & 47 & 39 & 33 & 26 & 200 \\
\hline Mean & 27.5 & 23.5 & 19.5 & 16.5 & 13.5 & \\
\hline
\end{tabular}

Table 5: Ethanolic extracted from Grape leaf zone of inhibition ( $\mathrm{mm}$ )

\begin{tabular}{|c|c|c|c|c|c|c|}
\hline \multirow{3}{*}{$\begin{array}{c}\text { Orga } \\
\text { nisms }\end{array}$} & $\mathrm{A}$ & $\mathrm{B}$ & $\mathrm{C}$ & $\mathrm{D}$ & $\mathrm{E}$ & \multirow{2}{*}{ Total } \\
& $(18 \mathrm{~m}$ & $(16 \mathrm{~m}$ & $(14 \mathrm{~m}$ & $(12 \mathrm{~m}$ & $(10 \mathrm{~m}$ & \\
& $\mathrm{g} / \mathrm{ml})$ & $\mathrm{g} / \mathrm{ml})$ & $\mathrm{g} / \mathrm{ml})$ & $\mathrm{g} / \mathrm{ml})$ & $\mathrm{g} / \mathrm{ml})$ & \\
\hline
\end{tabular}




\begin{tabular}{|c|c|c|c|c|c|c|}
\hline $\begin{array}{c}\text { Staph } \\
y . \\
\text { aureu } \\
s\end{array}$ & 23 & 20 & 18 & 15 & 10 & 86 \\
\hline $\begin{array}{c}E . \\
\text { coli }\end{array}$ & 13 & 9 & 0 & 0 & 0 & 22 \\
\hline Total & 36 & 29 & 18 & 15 & 10 & 108 \\
\hline Mean & 18 & 14.5 & 9 & 7.5 & 5 & \\
\hline
\end{tabular}

Table 6: The most significant treatment in each extracted were collated

Zone of inhibition (mm)

\begin{tabular}{|c|c|c|c|c|c|}
\hline \multirow{2}{*}{$\begin{array}{c}\text { Organis } \\
\text { ms }\end{array}$} & $\begin{array}{c}\text { S/Potato } \\
\text { Leaf } \\
\text { (Methan } \\
\text { ol) }\end{array}$ & $\begin{array}{c}\text { S/Potat } \\
\text { o Leaf } \\
\text { (Ethan } \\
\text { ol) }\end{array}$ & $\begin{array}{c}\text { Grape } \\
\text { Leaf } \\
\text { (Methan } \\
\text { ol) }\end{array}$ & $\begin{array}{c}\text { Grape } \\
\text { Leaf } \\
\text { Ethan } \\
\text { ol) }\end{array}$ & $\begin{array}{c}\text { Tot } \\
\text { al }\end{array}$ \\
\hline $\begin{array}{c}\text { Staphy. } \\
\text { aureus }\end{array}$ & 24 & 18 & 26 & 23 & 86 \\
\hline E. coli & 26 & 20 & 29 & 13 & 22 \\
\hline Total & 50 & 38 & 55 & 36 & 108 \\
\hline Mean & 25 & 19 & 27.5 & 18 & \\
\hline
\end{tabular}

\section{A. Statistical analysis}

The data collected was analyzed with the aid of Minitab and SPSS software and the following results were obtained

Table 7: Methanolic extracted from sweet Potato leaf Two-way ANOVA: observation versus Treatments, Organisms Source

\begin{tabular}{|c|c|c|c|c|c|}
\hline Source & DF & SS & MS & $\mathrm{F}$ & $\mathrm{P}$ \\
\hline Treatments & 4 & 227.0 & 56.75 & 42.04 & 0.002 \\
\hline Organisms & 1 & 1.6 & 1.60 & 1.19 & 0.338 \\
\hline Error & 4 & 5.4 & 1.35 & & \\
\hline Total & 9 & 234.0 & & & \\
\hline
\end{tabular}

$\mathrm{S}=1.162 \quad \mathrm{R}-\mathrm{Sq}=97.69 \% \quad \mathrm{R}-\mathrm{Sq}(\mathrm{adj})=94.81 \%$

FINDING: P-value under treatments $(0.002)<$ level of significant (0.05)

\section{Table 8:Post Hoc Tests}

Duncan $^{\mathrm{a}, \mathrm{b}}$

\begin{tabular}{|c|c|c|c|c|c|}
\hline \multirow{2}{*}{ Treatments } & & \multicolumn{4}{|c|}{ Subset } \\
\cline { 2 - 6 } & $\mathrm{N}$ & 1 & 2 & 3 & 4 \\
\hline E & 2 & 11.00 & & & \\
D & 2 & & 15.50 & & \\
C & 2 & & 17.50 & & \\
B & 2 & & & 21.00 & \\
& & & & & \\
A & 2 & & & & 25.00 \\
Sig. & & 1.000 & .160 & 1.000 & 1.000 \\
\hline
\end{tabular}

FINDING: Treatment $\mathrm{A}$ has the highest value in the subsets
Table 9: Ethanolic extracted from sweet Potato leaf

Two-way ANOVA: observation versus Treatments, Organisms

$\begin{array}{llllll}\text { Source } & \text { DF } & \text { SS } & \text { MS } & \text { F } & \text { P }\end{array}$

$\begin{array}{llllll}\text { Treatments } & 4 & 147.0 & 36.75 & 245.00 & 0.000\end{array}$

$\begin{array}{llllll}\text { Organisms } & 1 & 6.4 & 6.40 & 42.67 & 0.003\end{array}$

$\begin{array}{llll}\text { Error } & 4 & 0.6 & 0.15\end{array}$

Total $9 \quad 154.0$

$\mathrm{S}=0.3873 \quad \mathrm{R}-\mathrm{Sq}=99.61 \% \quad \mathrm{R}-\mathrm{Sq}(\operatorname{adj})=99.12 \%$

FINDING: P-value under treatments is < level of significant $(0.05)$

Table 10: Post Hoc Tests

Duncan $^{\mathbf{a}, \mathbf{b}}$

\begin{tabular}{|c|c|c|c|c|c|c|}
\hline \multirow{2}{*}{ Treatments } & & \multicolumn{5}{|c|}{ Subset } \\
\cline { 2 - 7 } & $\mathrm{N}$ & 1 & 2 & 3 & 4 & 5 \\
\hline E & 2 & 8.50 & & & & \\
D & 2 & & 11.00 & & & \\
C & 2 & & & 14.50 & & \\
B & 2 & & & & 17.00 & \\
A & 2 & & & & & 19.00 \\
Sig. & & 1.000 & 1.000 & 1.000 & 1.000 & 1.000 \\
\hline
\end{tabular}

FINDING: Treatment A has the highest value in the subsets

Table 11: Methanolic extracted from Grape leaf

Two-way ANOVA: observation versus Treatments, Organisms

Source DF SS MS F P

$\begin{array}{llllll}\text { Treatments } & 4 & 260.0 & 65.0 & 59.09 & 0.001\end{array}$

$\begin{array}{llllll}\text { Organisms } & 1 & 19.6 & 19.6 & 17.82 & 0.013\end{array}$

$\begin{array}{llll}\text { Error } & 4 & 4.4 & 1.1\end{array}$

Total 9284.0

$\mathrm{S}=1.049 \quad \mathrm{R}-\mathrm{Sq}=98.45 \% \quad \mathrm{R}-\mathrm{Sq}(\mathrm{adj})=96.51 \%$

FINDING: P-value under treatments is < level of significant (0.05)

Table 12: Post Hoc Tests

Duncan ${ }^{\mathrm{a}, \mathrm{b}}$

\begin{tabular}{|c|c|c|c|c|c|c|}
\hline \multirow{2}{*}{ Treatments } & & \multicolumn{5}{|c|}{ Subset } \\
\cline { 2 - 7 } & $\mathrm{N}$ & 1 & 2 & 3 & 4 & 5 \\
\hline E & 2 & 13.00 & & & & \\
\hline & & & & & & \\
D & 2 & & 16.50 & & & \\
C & 2 & & & 19.50 & & \\
B & 2 & & & & 23.50 & \\
A & 2 & & & & & 27.50 \\
Sig. & & 1.000 & 1.000 & 1.000 & 1.000 & 1.000 \\
\hline
\end{tabular}

FINDING: Treatment A has the highest value in the subsets 
Table 13: Ethanolic extracted from Grape leaf

Two-way ANOVA: observation versus Treatments, Organisms $\begin{array}{llllll}\text { Source } & \text { DF } & \text { SS } & \text { MS } & \text { F } & \text { P }\end{array}$ $\begin{array}{llllll}\text { Treatments } & 4 & 226.6 & 56.65 & 8.92 & 0.028\end{array}$ $\begin{array}{llllll}\text { Organisms } & 1 & 409.6 & 409.60 & 64.50 & 0.001\end{array}$ $\begin{array}{llll}\text { Error } & 4 & 25.4 & 6.35\end{array}$

Total 9661.6

$\mathrm{S}=2.520 \quad \mathrm{R}-\mathrm{Sq}=96.16 \% \quad \mathrm{R}-\mathrm{Sq}(\mathrm{adj})=91.36 \%$

FINDING: P-value under treatments is < level of significant (0.05)

Table 14: Post Hoc Tests

Duncan ${ }^{\mathrm{a}, \mathrm{b}}$

\begin{tabular}{|c|c|c|c|c|}
\hline \multirow{2}{*}{ Treatments } & \multicolumn{2}{|c|}{ Subset } \\
\cline { 2 - 5 } & $\mathrm{N}$ & 1 & 2 & 3 \\
\hline E & 2 & 5.00 & & \\
$\mathrm{D}$ & 2 & 7.50 & 7.50 & \\
$\mathrm{C}$ & 2 & 9.00 & 9.00 & \\
$\mathrm{~B}$ & 2 & & 14.50 & 14.50 \\
$\mathrm{~A}$ & 2 & & & 18.00 \\
Sig. & & .193 & .053 & .237 \\
\hline
\end{tabular}

FINDING: Treatment A has the highest value in the subsets

Table 15: Most significant treatment in each extracted Two-way ANOVA: observations versus Treatments, Organisms

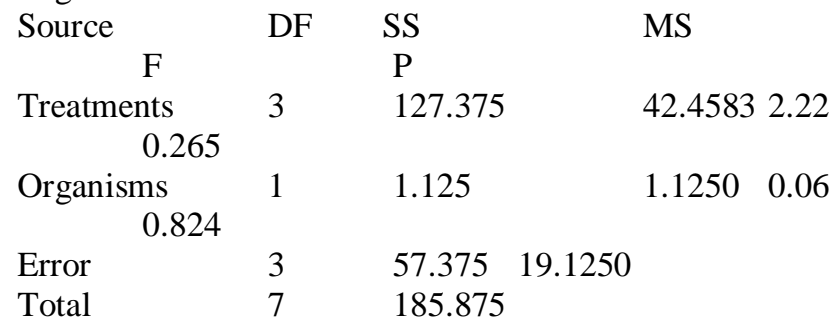

$$
\mathrm{S}=4.373 \mathrm{R}-\mathrm{SQ}=69.13 \% \mathrm{R}-\mathrm{SQ}(\mathrm{ADJ})=27.98 \%
$$

\section{DISCUSSION OF RESULTS}

From the table of result the followings were found:

The p-value of treatments in the table 7, 9, 11, and 13 were less than there corresponding level of significant (0.05), this indicates that significant difference exist between the treatments applied. Also, in table 8, treatment $\mathrm{C}$ and $\mathrm{D}$ were in the same subset column, meaning that difference exist between all the treatments except $\mathrm{C}$ and D. Treatments in table 10 and 12 were in separate subset column, meaning that difference exist between all the treatments. From table 14 significant difference only exist between treatment $\mathrm{AC}, \mathrm{AD}, \mathrm{AE}$ and $\mathrm{BE}$. Then treatment $A$ in table 8, 10, 12 and 14 has the highest value of comparison. But in determining which of these plants extract is highly significant, the most significant treatment (treatment A) in each plant extracted were compared and the p-value of treatments in the table 15 is greater that the level of significant
(0.05), this indicates that there is no significant difference among these plant extracted.

\section{CONCLUSION}

$18 \mathrm{mg} / \mathrm{ml}$ of the methanol and ethanol extracted from sweet potato and grape leaf were the best inhibiting the growth of these micro-organisms in this study. Therefore, any of these plants extracted at $18 \mathrm{mg} / \mathrm{ml}$ concentration were useful in preventing any of these micro-organisms.

\section{REFERENCE}

[1] Ali R., Mlambo V., Mangwe M.C., Dlamini, B.J.(2015) Chemical composition, nitrogen degradability and in vitro ruminal biological activity of tannins in vines harvested from four tropical sweet potato (Ipomoea batatas L.) varieties. Journal of Animal Physiology and Animal Nutrition, vol. 100, Pp. 61-68.

[2] Ben-Arye E.N, Samuels L.H., Goldstein K., Mutafoglu S., Omran, E. Schiff, H. Charalambous, T. Dweikat, I. Ghrayeb, G. Bar-Sela, I. Turker, A. Hassan, E. Hassan, B. Saad, O. Nimri, R. Kebudi and Silbermann M. (2016) Potential risks associated with traditional herbal medicine use in cancer care: A study of Middle Eastern oncology health care professionals. Cancer 122(4): Pp. 598-610.

[3] Brenes A., Viveros A., Chamorro S. and Arija I. (2016) Use of polyphenol-rich grape by-products in monogastric nutrition, a review Animal Feed Science Technology, Vol. 211, Pp.1-17.

[4] De Moura F.F, Millof A. and Boy E. (2015) Retention of pro vitamin A carotenoids in sample crops targeted for biofication in Africa: cassava, maize and sweet potato. Critical Reviews in Food Science and Nutrition, Vol. 55, Pp.1246-1269.

[5] EI- Sheikhia A.F. and Ray R.C. (2015) Potential impacts of bioprocessing of sweet potato: review Critical in Food Nutrition.

[6] Floulkes D, Hovell F.D. and Preston T.R. (1997) Sweet potato forage as cattle feed: voluntary intake and digestibility of mixtures of sweet potato forage and sugarcane, Tropical Animal Production, Vol. 3, Pp.140-144.

[7] Frye J.B.J, Hawkins G.E.J and Henderson H.B (1948) The value of winter pasture and sweet potato meal for lactating dairy cows, Journal of Dairy Science., Vol. 31, Pp.1215-1221.

[8] Joshi S.S., Kuszynski C.A. and Bagchi D. (2001) The cellular and molecular basis of health benefits of grape seed proanthocyanidin extract. Curr. Pharm Biotechnol., Vol. 2(2), Pp.187- 200.

[9] Li P., Shuren J., Wang Q., Qin M., Hou C. and Shen Y. (2017) Adding sweet potato vines improve the quality of rice straw silage, Animal Science Journal, Vol. 88, Pp. 625-632. 
[10] Nayaata O.Z., Dorward P.T. and Keatinge J.D.H. (2000) O'Neil MK. Availability and use of dry season feed resources on small holder dairy farms in central Kenya. Agroforestry System, Vol. 50, Pp. 315331.

[11] Nowshehri J.A., Bhat Z.A. and Shah M.Y. (2015) Blessings in disguise: Bio-functional benefits of grape seed extracts. Food Res. Int., Vol. 77(3), Pp. 333-348.

[12] Rusoff L.L., Miller G.D., Burch B.L. and Frye J.B. (1950) Dehydrated sweet potatoes as a substitute for corn-soyabean silage, Journal of Dairy Science, Vol. 33, Pp. 657-660.

[13] Weseler A.R. and Bast A.( 2017) Masquelier's grape seed extract: from basic flavonoid research to a well characterized food supplement with health benefits, Nutritional Journal,Vol. 16(1), Pp. 5.

[14] Yahaya H, Gumel S.M,Musa H. and Ado H (2015)Ecofriendly dyeing of cotton and polyester fabrics with natural dyes extracted from different varieties of Kola nuts, international Journal of Chemical Sciences, Vol.1,Issue 1,Pp:6-11 